\title{
Diseño de Información como eje de un modelo para planear experiencias educativas digitales en medicina
}

\section{Information Design as the axis of a model to plan digital educational experiences in medicine}

\author{
Gerardo Luna-Gijón \\ gerardo.lunag@correo.buap.mx \\ Benemérita Universidad \\ Autónoma de Puebla \\ Puebla, Puebla, México \\ ORCID: 0000-0001-8216-767X
}

Recibido: 21 de marzo de 2021 Aprobado: 04 de junio de 2021 Publicado: 01 de julio de 2021

Resumen

Los principios disciplinares del Diseño de Información pueden ampliar las posibilidades de la experiencia educativa en la enseñanza médica. Este artículo muestra evidencia cualitativa de que un modelo en el que intervienen tres áreas distintas: medicina, educación y diseño, puede ser potenciado mediante la intervención de ideas y prácticas que prevalecen en el Diseño de Información como eje rector en la toma de decisiones, y como fuerza integradora de los procesos conceptuales y creativos, capaz de coordinar e integrar las diferentes perspectivas de estas tres áreas del conocimiento. Sumando el uso de métodos centrados en el usuario, como la técnica Delphi, se pudo abordar la creación de un modelo instruccional original que sirve como guía a los profesores de medicina para diseñar materiales digitales y fomentar el razonamiento clínico en estudiantes de pregrado.

Palabras clave: Diseño de Información; modelo instruccional; estudio cualitativo; educación digital en medicina; diseño de experiencias de aprendizaje.

\section{Abstract}

The disciplinary principles of Information Design can expand the possibilities of the educational experience in medical teaching. This article shows qualitative evidence that a model in which three different areas intervene: medicine, education and design, can be enhanced through the intervention of ideas and practices that prevail in Information Design as the guiding axis in decision-making, and as antegrating force of conceptual and creative processes, capable of coordinating and integrating the different perspectives of these three areas of knowledge. Adding the use of user-centered methods, such as the Delphi technique, it was possible to address the creation of an original instructional model that serves as a guide for medical teachers to design digital materials and promote clinical reasoning in undergraduate students.

Keywords: Information Design; instructional model; Qualitative study; digital education in medicine; design of learning experiences. 


\section{Introducción}

约 omo resultado de un proyecto de investigación realizado en 2014, se obtuvo el Modelo Instruccional para Diseñar Materiales Educativos Digitales para Medicina (MIDIMED) (Luna-Gijón y Porras-Hernández, 2014), el cual plantea una guía para elaborar secuencias didácticas aprovechando el entorno digital, empoderando la educación médica para estudiantes de pregrado.

Tomando en cuenta la situación generada a partir de la pandemia por COVID-19, se requiere revisar estrategias que potencien la educación en línea de calidad para los estudiantes, brindando al docente herramientas especializadas para diseñar y crear ambientes educativos enriquecidos, y otorgando a la comunidad de especialistas otros puntos de vista que amplíen la discusión sobre estos temas.

La intención de este artículo es dar una visión profunda en relación con la creación y el fundamento del MIDIMED, dando relevancia al papel de los principios y las estrategias del Diseño de Información (DI), pues el artículo original donde se presentó al midimed (Luna-Gijón y Porras-Hernández, 2014) aportó una visión global del proyecto y su validación, pero no dio suficiente atención para explicar el desarrollo del modelo ni la trascendencia que tuvo el DI en su creación.

El desarrollo conceptual del MIDIMED es relevante por lo que ofrece a la comunidad del diseño, al dar un panorama que contribuye a entender el desarrollo de modelos de diseño, al mostrar lo que esta área de conocimiento brinda al crecimiento de otras disciplinas y, en particular, al enriquecer la discusión sobre la aportación del DI al campo disciplinar y su acción mediante sus principios y estrategias. En cuanto a los docentes de la comunidad médica, les proporciona una profundidad y un sustento para generar un aprendizaje notable usando medios digitales.

Para el desarrollo del modelo se utilizó la técnica Delphi, buscando el consenso entre un grupo de expertos, a partir de una serie de rondas de evaluación del MIDMED. Con base en los comentarios realizados, se ha hecho un análisis cualitativo interpretativo del impacto del DI en el modelo.

En el presente documento se profundiza cómo se hizo el modelo, el proceso conceptual que le dio forma, así como los principios y la importancia 
del DI para que dicho modelo tenga un fundamento tanto en su aplicación para la educación médica como en su discusión para el diseño.

Antecedentes
Las tecnologías de la información influyen en las experiencias humanas de todos los días, al estar incrustadas en el ámbito de la casa, la escuela y el trabajo. Éstas transforman los modos de comunicarnos y nuestra cultura (Mendoza-Villamar y Quiroz-Valencia, 2019; Udsen y Jørgensen, 2005), lo que resulta en nuevas formas de conocimiento creadas por las experiencias digitales, un acercamiento personalizado hacia el usuario y sus aspectos cognitivos, y el entrelazado entre educación, tecnología y nuestra humanidad (Martínez-Fernández y Mahauad-Burneo, 2018).

Como consecuencia de ello, los ambientes de aprendizaje digitales son usados para generar experiencias educativas (Liang y Sedig, 2009; Real-Torres, 2019). Sin embargo, el diseñar un material efectivo puede ser una tarea complicada, pues los profesores deben entender cómo organizar la información que se presentará y adecuarla de manera que apoye la estructura del curso. Es decir, se requiere de un acto de planeación que extraiga y traduzca la información en un formato que enfatice el proceso de enseñanza (O'Connor, 2010), lo que convierte al diseño en pieza fundamental de la educación (Lin, 2005).

Elaborar, bajo las ideas del diseño, un material de aprendizaje digital, que puede ser desde la sencilla presentación mediante diapositivas digitales hasta un sofisticado simulador virtual, siempre tendrá como objetivo el capturar y motivar la atención del alumnado para presentar los puntos que el profesor quiere comunicar (Gallardo-Fernández, Castro-Calvo y Saiz-Fernández, 2020; O’Connor, 2010), de tal manera que los participantes se sumerjan en la experiencia de aprendizaje, relacionándose con la información narrada en incidentes tomados de la vida real, en una forma ordenada en la pantalla, donde las situaciones y soluciones planeadas se conviertan en algo integrado a su inventario de memorias retenidas de la experiencia real (Eisner, 2004; Ricardo-Barreto, Iriarte-Díazgranados, Said-Hung, Ballesteros-Cantillo, Jabba-Molinares, Manotas-Salcedo, Salas-Álvarez, Peláez-Cárdenas, Villa-Agudelo, Zapata-Álvarez, Aarón-Gonzálvez, Choles-Almazo, Ordoñez-Villa, Vergara-Castilla, Chavarro-Jiménez y Astorga-Acevedo, 2017).

De esta manera, se vislumbró la oportunidad de desarrollar un modelo instruccional que incorpore ideas de diversos campos del diseño (gráfico, instruccional, de interacción, de información), que ayude al profesor de medicina en su práctica educativa en el diseño y desarrollo de materiales educativos digitales, que sean usados como material didáctico en los cursos de preclínica.

Para elaborar este modelo se decidió un acercamiento que permitiera abarcar los procesos mentales donde se pueden establecer estilos 
cognitivos, es decir, patrones de pensamiento o de organización para ser usados en gran cantidad de situaciones y en numerosos tipos de información (Cárdenas-Figueroa, 2019; González Di Pierro, 2016), pasando por consideraciones propias de la era digital, donde se necesita enfocarse en las conexiones entre los elementos de diseño: medios, palabras, imágenes, movimiento y sonidos (Tarkhova, Tarkhov, Nafikov, Akhmetyanov, Gusev y Akhmarov, 2020; Wurman, 2001), hasta lograr englobar a los principales actores involucrados en la enseñanza: las personas, la escuela y el potencial para aprender (Bransford, Brown y Cocking, 2000; Miguel-Román, 2020).

A partir de esto, nació el MIDIMED, un producto original que busca desarrollar materiales de aprendizaje para la formación de los estudiantes de medicina, y que integra tres áreas del conocimiento: medicina, educación y diseño (véase figura 1 ).

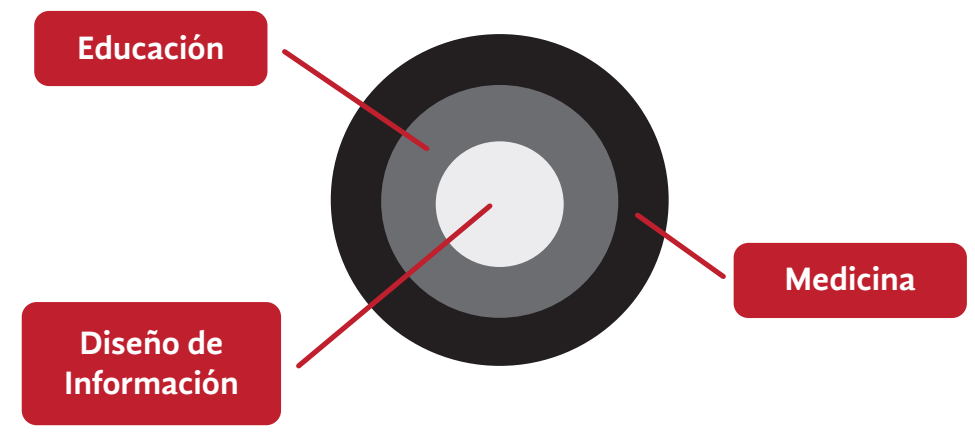

Figura 1. El modelo abarca tres temas centrales.

Fuente: Elaboración propia.

El MIDIMED está dirigido a los profesores médicos universitarios y opera suscrito a la idea de darle al estudiante la oportunidad de reflexionar, ejercitar y madurar el razonamiento clínico, desarrollando su capacidad de toma de decisiones al emplear la narrativa de casos clínicos, los cuales se presentan en formato digital, todo esto mediante un proceso donde se diseña la experiencia en pantalla y en donde el aprendiz puede madurar sus competencias en un entorno amigable y dinámico, con lo cual se espera resulten profesionistas médicos mejor preparados y capacitados para la atención al paciente. En este punto, el DI juega un papel principal al ser el conducto que une los datos que se presentan con la estructura visual final que ve el estudiante.

En cuanto a la metodología ocupada, se analizó la literatura especializada y reconocida sobre DI, de la cual se extrajeron las ideas persistentes, así como aquellas que tuvieran resonancia en la práctica, y finalmente se incorporaron ideas propias del autor que han sido maduradas por su experiencia académica y su práctica profesional. 
Por otro lado, se rescató el uso de la narrativa o storytelling como una de las herramientas más populares del diseño, aunque también sea una de las menos entendidas, pues mucho de lo que se dice y escribe sobre sus beneficios se basa en observaciones desde la práctica, más que en resultados de investigación (Agosto, 2016), lo que lleva a que aún exista poco entendimiento sobre qué factores predominan y cómo estos pueden ser combinados para crear un efectivo flujo en la narrativa, especialmente en aquella que se presenta visualmente (McKenna, Henry Riche, Lee, Boy y Meyer, 2017).

Para que desde el diseño se puedan responder estas cuestiones y sea posible alcanzar una implementación efectiva del modelo, es necesario reconocer que cualquier objeto, incluso uno visual, está constituido por un discurso, en el cual subyacen estructuras narrativas que lo pueden hacer más eficiente o menos interesante, dependiendo de qué tan bien esté identificada y elaborada dicha estructura en lo visual (Barnes, 2016). A partir de este reconocimiento es donde se empieza a trabajar con la narrativa, la cual consiste en «telling a story from memory without the aid of a book or written script» [contar una historia desde la memoria sin la ayuda de un libro o un guion escrito] (traducción propia) (Agosto, 2016, p. 21).

Con la comprensión de estos conceptos (narrativa, DI, medicina, educación y ambientes digitales) es que surgió la oportunidad del desarrollo del MIDIMED. El planteamiento inicial fue que en cualquier tipo de proyecto - ya fuera uno muy sencillo que tardara algunas horas en resolverse u otro complicado en su ejecución, que requiriera un trabajo de meses-, todos los diferentes procesos y las etapas que implicaba no eran otra cosa que información, la cual se encontraba en distintos estadios, ya fuera como datos burdos o como conocimiento complejo. Ésta debía ser organizada, transformada y llevada a una ejecución práctica en niveles variados, es decir, se realizaba un ejercicio de DI. En la figura 2 se muestran las cinco etapas del MIDIMED.

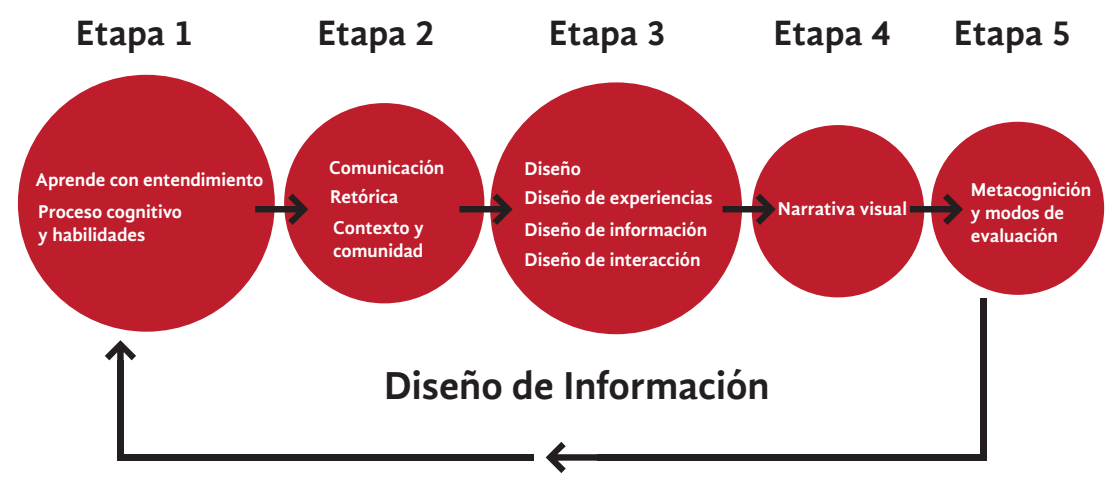

Figura 2. En las etapas actúan secuencialmente diferentes temas para producir una experiencia de diseño exitosa.

Fuente: Elaboración propia. 
Al reconocer que la cantidad de conocimiento perteneciente al área médica se incrementa exponencialmente día a día, el MIDIMED busca apoyar al docente que considere que mucho de su esfuerzo debe de enfocarse en una eficiencia en la presentación digital de la información (Bove, 2008; S. C. Hautz, Hoffmann, Exadaktylos, W. E. Hautz y Sauter, 2020), de manera que el proceso de razonamiento del estudiante se vea potenciado por el medio electrónico.

Por otro lado, si se reconoce que las bases científicas de la medicina radican en las ciencias naturales, pero que la ejecución clínica también usa métodos y conceptos de las ciencias sociales (Boye, Eberholst, Farlie, Sørensen y Lyng, 2007) y que la práctica misma con el paciente se nutre del humanismo, se comprenderá que el diseño como disciplina funge como un punto de convergencia de las ciencias sociales y el humanismo, por lo que conforma un área de acción que puede aportar recursos valiosos a la medicina respecto al diseño de materiales educativos digitales. Esta consideración llevó a realizar una revisión amplia de la literatura pertinente a estos temas. De ahí que, en la figura 3 , se aprecie la matriz conceptual que une autores con conceptos clave del di para elaborar el proyecto.

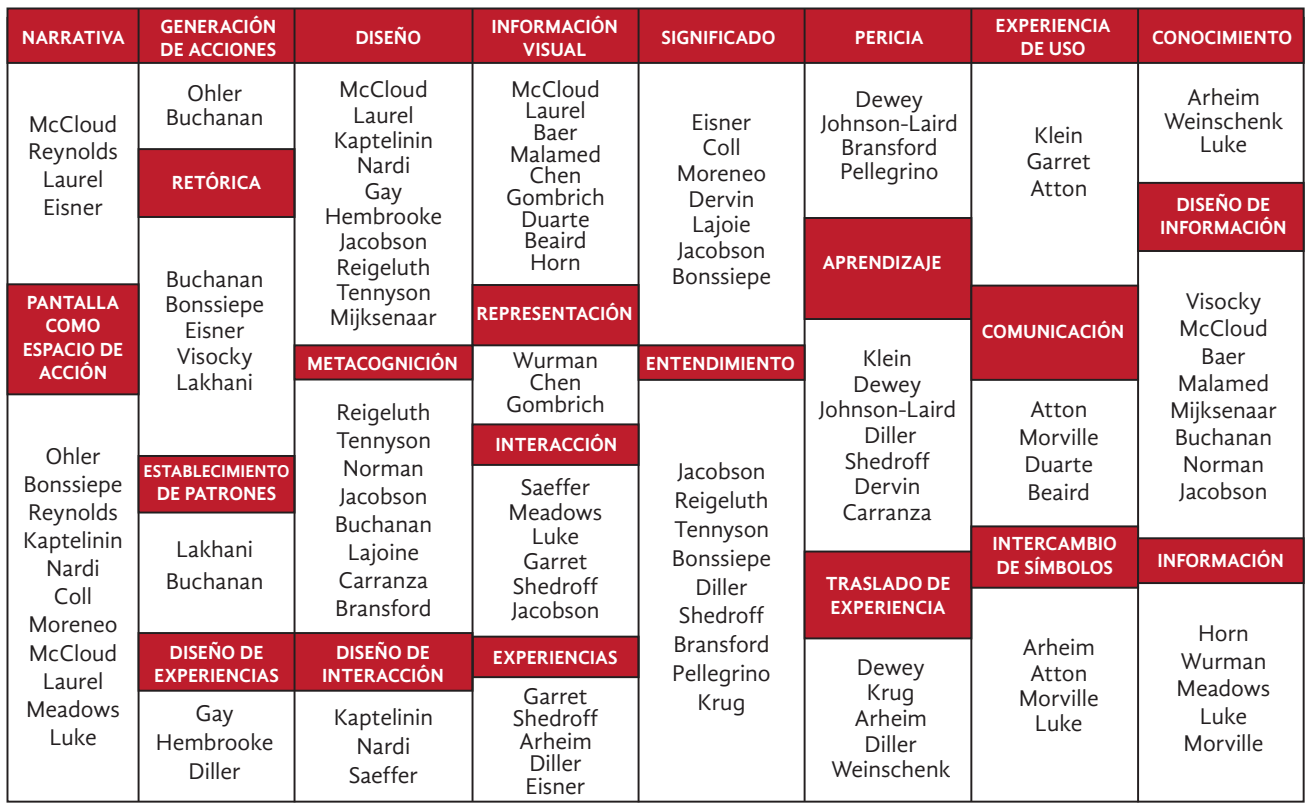

Figura 3. Matriz conceptual donde se vinculan autores y conceptos clave del DI. Fuente: Elaboración propia.

Pero ¿cómo se conjugan en un mismo material digital las disciplinas de medicina, educación y diseño?, ¿cómo elegir el camino apropiado para lidiar con la información compleja resultado de la acción de estas mismas?

El Diseño de Información se encarga de encontrar la estrategia y de organizar cómo presentar información, para que ésta sea fácil de entender y usar de forma efectiva y eficiente, con el fin de que las personas pueden tomar decisiones (Horn, 2000; Uribe, 2019). 
La naturaleza del DI es buscar dar sentido a las cosas, lo que se alinea perfectamente con un modelo educativo constructivista, y con las necesidades de los profesores para ser facilitadores del conocimiento.

Complementando esto, tenemos las ideas de Horn (2000) sobre cuáles son los objetivos primarios del DI:

1. Desarrollar documentos que son comprensibles, certera y rápidamente recuperables y con facilidad de convertirlos en acción efectiva.

2. Diseñar interacciones que son naturales, sencillas y lo más placenteras posibles.

3. Permitir a las personas encontrar su camino en diversos ambientes con comodidad y facilidad.

4. Permitir un proceso de comunicación con eficiencia y efectividad.

La relevancia de estas ideas se pondera cuando consideramos las siguientes condiciones (Visocky O'Grady y Visocky O'Grady, 2008):

1. Vivimos en un mundo cada vez más complejo, en donde la necesidad de una comunicación clara y accesible, a través de una amplia gama de medios, se ha vuelto esencial.

2. Existe una demanda para que el conocimiento sea más accesible, la cual se incrementa con el devenir del ritmo del mundo moderno.

Pontis (2019) señala al DI como una vía para solucionar lo anterior debido a que:

1. El DI logra sus metas al combinar visualizaciones, ilustración, estudios de comunicación, ergonomía, psicología, sociología, lingüística, antropología, ciencias de la computación y una variedad de otros campos.

2. El DI se enfoca en la representación precisa de cuerpos de conocimiento y las necesidades específicas del usuario final que se acerca a ese conocimiento.

3. El DI coloca al usuario en el centro del proceso de diseño.

Además de lo anterior, para personalizar, robustecer y dar una aportación, es posible añadir otras ideas extraídas de la experiencia del autor y que se pueden considerar principios del DI:

1. Hacer DI significa que la información debe llevar a las personas al entendimiento.

2. El entendimiento lleva a las personas a tomar acción en su vida.

3. El tomar acción lleva al empoderamiento.

4. El DI es una puerta que permite a las personas interactuar con el conocimiento. 
El objetivo es aplicar estas ideas en el modelo MIDIMED, que se centra en el desarrollo de materiales educativos que se visualizan a través de un medio particular; así, la pantalla se convierte en una herramienta pedagógica poderosa, que sirve para desarrollar las capacidades humanas y los contextos sociales para el aprendizaje. Esto puede ser alcanzado con éxito si se logra involucrar a las personas de tal manera que, al interactuar con la información presentada, tengan una experiencia envolvente y enriquecedora. Así, el profesor debe estar consciente de la importancia e impacto que ésta tiene sobre la experiencia del estudiante.

La pantalla es mucho más que un dispositivo pasivo, es una herramienta con un gran potencial que vincula y permite la interacción de la información con nuestra mente, por lo cual se considera que, más que una ventana, la proyección de aquello que vemos en la pantalla es una puerta. Una ventana nos permite ver el panorama de diferentes maneras, pero es una forma muy sencilla de interacción que sitúa al ser humano como observador; en cambio, una puerta nos permite un discurso con mayor dinamismo, en el que, además de las distintas vistas, podemos entrar y salir y, sobre todo, introducir y retirar artículos, reorganizar la disposición entre ellos y el otro espacio, es decir, transportar cosas, en este caso, ideas y conocimiento.

\section{El DI como una guía invisible para el proyecto}

El sólo llenar de información a las personas no tiene sentido. La forma en cómo ésta se presenta y se organiza es tan importante como el contenido. Así, se vuelven prioritarios los procesos cognitivos (Uribe, 2019): encontrar, escudriñar, ordenar, organizar, memorizar, señalar y atender a la información. La integración de todo esto permite que los consumidores finales elijan la opción más adecuada para tener una experiencia (Wurman, 2001). Por ello y porque el contenido y los materiales descriptivos serán reunidos por los usuarios (Real-Torres, 2019), es que los diversos objetos digitales deben ser contextualizados apropiadamente, dentro de este colectivo virtual de ideas y experiencias.

Este contexto común, que permite a las personas ordenar conceptualmente y entender aquello que están percibiendo, puede ser construido mediante dos herramientas que usa el DI:

1. Narrativa

2. Retórica visual

La narrativa es importante para el DI porque parece algo contrario a la naturaleza humana el separar cualquier información del mundo en que esa información existe (Figueiredo, 2011). El narrar historias está profundamente arraigado al comportamiento social de los grupos humanos. Las historias son usadas para educar y enseñar dentro de la comunidad, discutir valores y moral, o para satisfacer la curiosidad. En ellas 
se dramatizan las relaciones sociales y los problemas de la vida al transmitir ideas o plantear fantasías (Eisner, 2004).

Por su parte, la retórica es un recurso imprescindible porque permite establecer un discurso en donde se muestran opciones a las personas y la manera en que éste se presenta va de acuerdo con los estilos cognitivos, al tomar en cuenta los diferentes modos de aprendizaje del ser humano. De esto trata la retórica: de convencer a las personas dando la mejor opción y argumentando en relación con el perfil específico de la persona con la que nos estamos comunicando.

\section{DI y constructivismo}

El MIDIMED es un proyecto educativo que busca mejorar la calidad de la enseñanza médica, entonces comenzaremos a establecer los vínculos con la teoría educativa en que se encuentra inserto el proyecto: el constructivismo. Éste se basa en la idea de que el aprendizaje es un proceso activo de construcción del conocimiento, que se da a partir de lo que sabemos y que, al no ser un proceso pasivo de transmisión o un despliegue de conocimientos innatos, nos permite crear experiencias al acomodar y reorganizar nuestras estructuras mentales (Pavlović y Maksić, 2019; Serrano y Pons, 2011; Solomonidou, 2009).

A partir de lo anterior, se pueden establecer parecidos entre el DI y la teoría del constructivismo:

1. Edificación es el proceso de iluminación personal (Jacobson, 2000) que claramente está en línea con la idea de que las personas construyen su conocimiento.

2. Conmutatividad es el proceso de cambio mutuo (Jacobson, 2000), podemos verlo como una revisión de que el individuo que construye conocimiento no lo hace solo, sino que el mejor aprendizaje se da en la compañía y con la mediación de otras personas.

3. La información debe ser accesible (Wurman, 2001) o, dicho de otra forma: «El conocimiento debe ser para todos». Además, se intuye que también quiere decir que todas las personas tenemos la capacidad para aprender.

4. Se aprende cuando relacionamos la información con algo que entendemos (Wurman, 2001). Esto se vincula con la idea de que los humanos modificamos nuestro conocimiento a partir de tener en claro ideas fundamentales que podemos expandir, transferir y adaptar.

5. Aprender significa hacer conexiones (Wurman, 2001), es decir, el aprender con significado permite transferir el conocimiento hacia otras situaciones, lo que, a su vez, permite el desarrollo de nuestras habilidades y competencias.

6. El entendimiento, la acción, y el empoderamiento, como ideas del $\mathrm{DI}$, equivalen a que seamos capaces de responder a las preguntas: 
¿qué es?, ¿cómo se hace?, ¿para qué sirve? y ¿cuándo debe ser utilizado?

7. La pantalla de los dispositivos digitales, como una puerta que nos permite acceder y manipular productos del conocimiento, se puede entender como parte de uno de los máximos alcances de nuestra capacidad de aprendizaje: la metacognición, que, de acuerdo con Bransford et al. (2000), significa ser conscientes de nuestro aprendizaje y poder evaluar nuestro desempeño, reflexionando sobre lo que hacemos.

La teoría revisada permite comprender al Dı y a los principios de éste que se consideraron valiosos en la generación del modelo, lo cual ofrece un panorama con el que se puede revisar la interacción y la acción del DI, así como su actuación subyacente al aplicar las etapas del MIDIMED. En la figura 4 se aprecia un mapa conceptual donde se muestran los temas clave del MIDIMED y la relación que hay entre ellos.

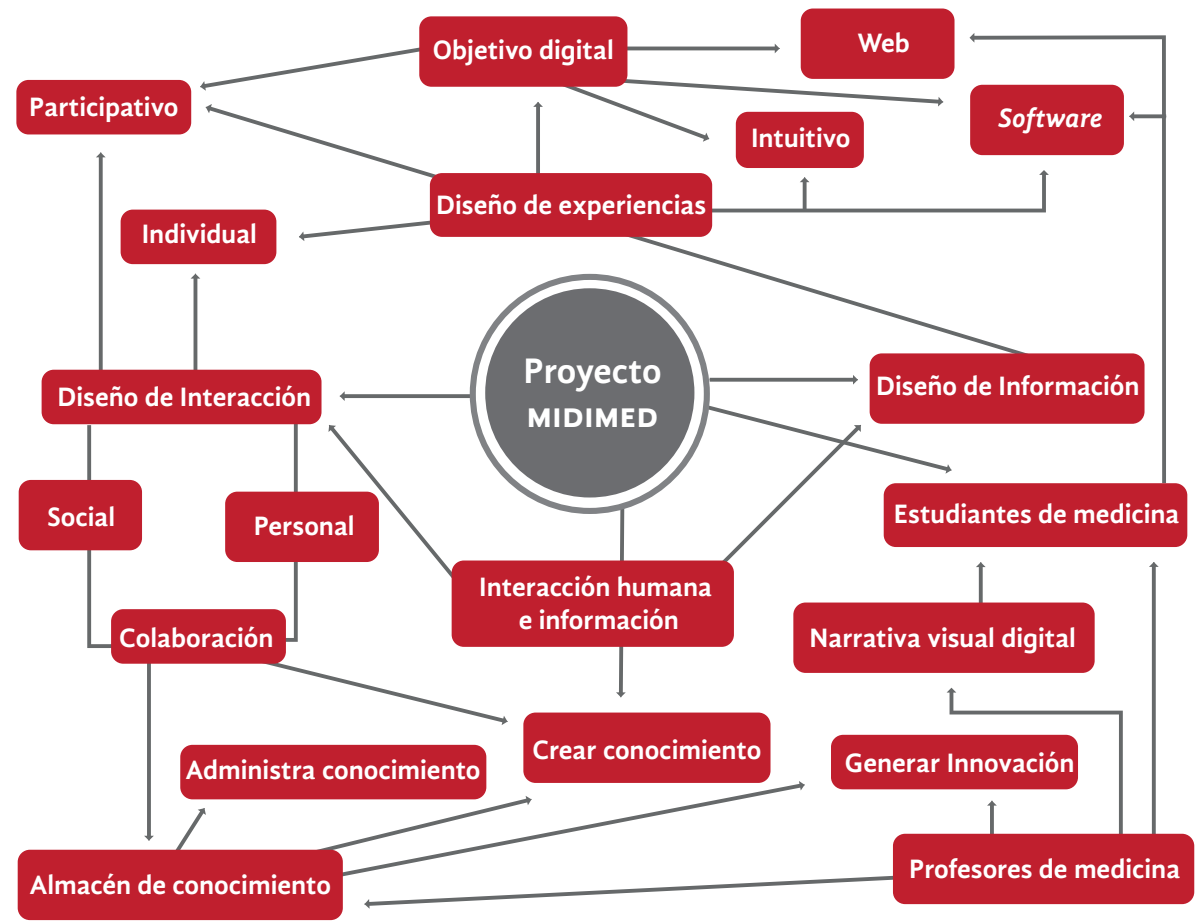

Figura 4. Mapa conceptual con las ideas principales del DI que se conjugan en el MIDIMED.

Fuente: Elaboración propia.

Método Como hablamos de una experiencia y de cómo se está produciendo el conocimiento, esto nos inserta en un proceso cualitativo, porque se trabaja de forma sistemática a través de un método flexible - donde el investigador se adentra en entender las situaciones, generando vínculos con los 
participantes y logrando una profundidad del contexto- y orientado a los valores (Pontis, 2019).

El modelo fue validado por un grupo de profesores expertos pertenecientes a tres áreas del conocimiento, mediante una serie de rondas y el empleo de la técnica Delphi. El propósito de esta técnica cualitativa es probar la opinión y buscar el consenso de un tema entre un grupo de expertos provenientes de diferentes áreas de especialidad (Xu, Zare, Dai, Xiang y Gaskin, 2019). Al respecto, consenso se puede definir como el punto de vista aceptable por la mayoría (Ab Latif, Dahlan, Ab Mulud y Mat Nor, 2017; Vernon, 2009). También fue realizada una prueba piloto, donde un profesor siguió las etapas y los pasos hasta obtener un material digital para su clase (véase figura 5).

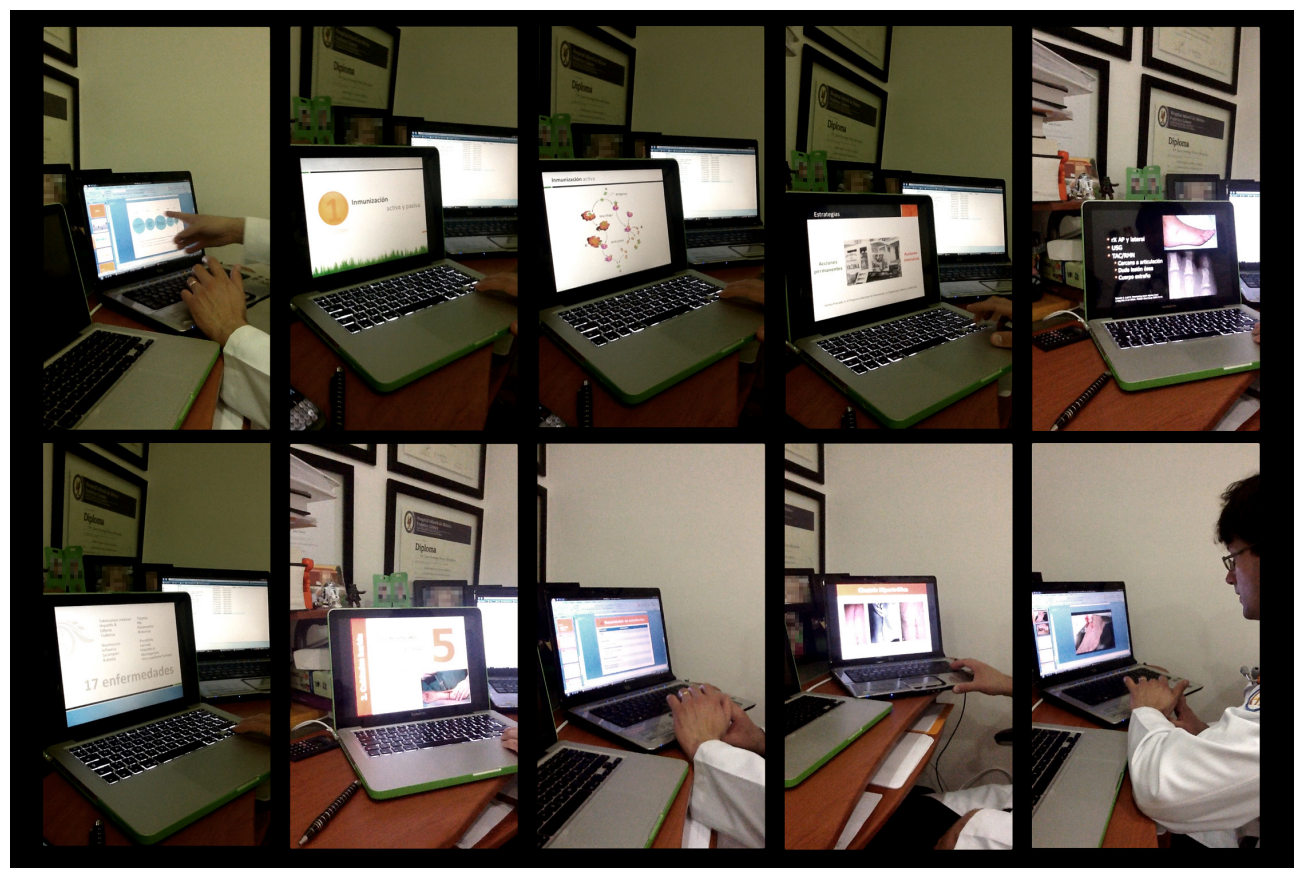

Figura 5. El docente explica su experiencia al usar el midimed para diseñar su material digital.

Fuente: Elaboración propia basada en un registro fotográfico personal.

Para la evaluación del MIDIMED se buscaron expertos, los cuales se pueden definir como personas que tienen un conocimiento profundo sobre el tópico o tema en cuestión, ya que pueden identificar características y patrones significativos que representan el punto de vista de su área y de sus colegas, y pensar reflexivamente acerca de los problemas de su disciplina (Bransford et al., 2000; Xu et al., 2019). Estos expertos debían cumplir con el siguiente perfil: tener experiencia práctica en su disciplina, estar inmersos en un área de conocimiento afín al proyecto (medicina, educación, diseño) y contar con pericia en el uso de materiales digitales. Para más información sobre las rondas, o acerca de las características y la aportación de los expertos se puede consultar el artículo 
publicado anteriormente (Luna-Gijón y Porras-Hernández, 2014), ya que en el presente escrito sólo se destaca la aportación cualitativa del DI para el MIDIMED.

En resumen, a partir de las aportaciones de los expertos, las bases teóricas del proyecto y la experiencia profesional, se desarrolló el proceso conceptual y práctico para establecer las etapas del modelo, las cuales fueron refinándose mediante las rondas de investigación producidas con la técnica Delphi, y se culminó el proceso con la prueba piloto con el docente.

Resultados El MIDIMED consta de cinco etapas: proyecta, comunica, estructura, narra, evalúa. El retomar principios del DI al construir el modelo permitió enriquecer los contenidos de estas etapas. En la tabla 1 se pueden ver los vestigios de la acción del di en cada una de ellas. La primera columna corresponde a cada etapa y lo que se debe hacer en ella (recordando que la etapa es el resultado de la combinación de tres áreas: educación, medicina y la conjunción de varios tipos de diseño), mientras que la segunda columna muestra un filtro realizado por el autor, a partir de la retroalimentación de los participantes, el cual permite observar los principios y las estrategias originales del di que dan fundamento a la etapa, y que quedan ocultos actuando de manera subyacente debido al conjunto (por esto se le ha denominado Eco del DI).

Tabla 1. El Di en las etapas del Midimed

\begin{tabular}{|c|c|}
\hline \multicolumn{2}{|c|}{ Etapa 1} \\
\hline Objetivo & Eco del DI \\
\hline $\begin{array}{l}\text { En esta etapa se busca enfatizar y } \\
\text { sensibilizar al profesor para que consi- } \\
\text { dere la planeación de sus clases en } \\
\text { función del aprendizaje y desarrollo } \\
\text { de competencias del estudiante, que } \\
\text { es el centro de su quehacer docente. Al } \\
\text { conocer a su estudiante e identificar } \\
\text { sus fortalezas y necesidades, el profe- } \\
\text { sor puede establecer las estrategias y } \\
\text { metas de instrucción al desarrollar el } \\
\text { curso. }\end{array}$ & $\begin{array}{l}\text { Los contenidos enfatizan la idea del DI } \\
\text { sobre apoyar el entendimiento, tomar } \\
\text { en cuenta a las personas y sus formas } \\
\text { de aprender; que el entendimiento } \\
\text { lleve a la acción y, con esto, al desarro- } \\
\text { llo y a la mejora de las habilidades en la } \\
\text { persona, es decir, se enfoca en fortale- } \\
\text { cer la edificación y la conmutatividad. }\end{array}$ \\
\hline
\end{tabular}




\begin{tabular}{|c|c|}
\hline \multicolumn{2}{|c|}{ Etapa 2} \\
\hline Objetivo & Eco del DI \\
\hline $\begin{array}{l}\text { Esta etapa se enfoca en entender } \\
\text { cómo comunicar de mejor manera la } \\
\text { instrucción y los contenidos concep- } \\
\text { tuales, procedimentales y actitudinales } \\
\text { por aprender, al definir las estrategias } \\
\text { con las que el profesor establecerá una } \\
\text { comunicación efectiva con el fin de } \\
\text { hacer más entendible y accesible el } \\
\text { aprendizaje para el estudiante. }\end{array}$ & $\begin{array}{l}\text { Mediante las ideas del DI, este proceso } \\
\text { de comunicación significa facilitar al } \\
\text { acceso a la información, de manera } \\
\text { que el aprender y generar conocimien- } \\
\text { to sea posible para todos los miembros } \\
\text { de la clase. Esto sólo es posible cuando } \\
\text { los medios y documentos que intervie- } \\
\text { nen son comprensibles, ya que serán } \\
\text { diseñados de acuerdo con las necesida- } \\
\text { des específicas del usuario final. Como } \\
\text { todo sucede en la pantalla de un dispo- } \\
\text { sitivo, se comienza a trabajar la idea de } \\
\text { que el medio digital actúa como una } \\
\text { puerta que facilita la interacción de la } \\
\text { información con nuestra mente. }\end{array}$ \\
\hline
\end{tabular}

\begin{tabular}{|c|c|}
\hline \multicolumn{2}{|c|}{ Etapa 3} \\
\hline Objetivo & Eco del DI \\
\hline $\begin{array}{l}\text { Planear, mediante el diseño, la estrate- } \\
\text { gia, la estructura, las representaciones, } \\
\text { las experiencias y los elementos visua- } \\
\text { les que conforman el producto final. En } \\
\text { esta etapa se busca que el profesor } \\
\text { identifique al diseño como un área } \\
\text { necesaria para apoyarse y dar forma a } \\
\text { la construcción del material digital. }\end{array}$ & $\begin{array}{l}\text { Se enfatiza que, través de los elemen- } \\
\text { tos del diseño visual, el DI va a lograr } \\
\text { acercar el conocimiento tomando } \\
\text { como centro a las personas, en un } \\
\text { ambiente seguro y donde cada quien } \\
\text { puede aprender a su ritmo. }\end{array}$ \\
\hline \multicolumn{2}{|c|}{ Etapa 4} \\
\hline Objetivo & Eco del DI \\
\hline $\begin{array}{l}\text { En esta etapa, el objetivo es generar la } \\
\text { forma definitiva del producto final. Se } \\
\text { busca que el profesor termine el mate- } \\
\text { rial digital al establecer el camino del } \\
\text { caso clínico que recorrerán los estu- } \\
\text { diantes, determinar la entrada y las } \\
\text { posibles salidas de ese camino, y esta- } \\
\text { blecer cómo se combinan los puntos } \\
\text { de vista o medios involucrados. }\end{array}$ & $\begin{array}{l}\text { La intervención de las dos herramientas } \\
\text { más poderosas del di, narrativa y retóri- } \\
\text { ca, sirven para ejercer de guías y víncu- } \\
\text { los que ayuden al estudiante a moverse } \\
\text { por la puerta que significa el medio } \\
\text { digital, y a encaminar sus pasos de un } \\
\text { espacio a otro, de manera que se } \\
\text { logren generar objetos de conocimien- } \\
\text { to en el acto de aprender. Así, se sigue } \\
\text { con la idea de que la reunión de aquello } \\
\text { que vemos en pantalla es una puerta } \\
\text { que permite un acceso dinámico al } \\
\text { conocimiento. }\end{array}$ \\
\hline
\end{tabular}




\section{Etapa 5}

En esta etapa se pretende que el profesor establezca los puntos y niveles específicos que busca evaluar durante el curso. Al final es importante considerar cómo se evaluará el impacto del material digital diseñado, tanto a nivel del estudiante, como de la relación profesor-alumno y profesor-grupo.
Se vuelve a la idea de comunidad, edificación y conmutatividad, pero reforzadas con el propósito de verificar si se ha llegado al entendimiento $y$, con esto, a una transformación del individuo, en su conocimiento, debido a la información, al adquirir nuevas habilidades y proficiencia en su actuar, logrando con esto un empoderamiento de la persona humana.

Fuente: Elaboración propia

Señalar estos ecos hace visible la acción del DI (véase figura 6), que tiene un impacto en cada momento del modelo, de tal manera, que son interpretados a partir de un proceso dialéctico cualitativo que toma como referencia el marco teórico y la experiencia del proceso.

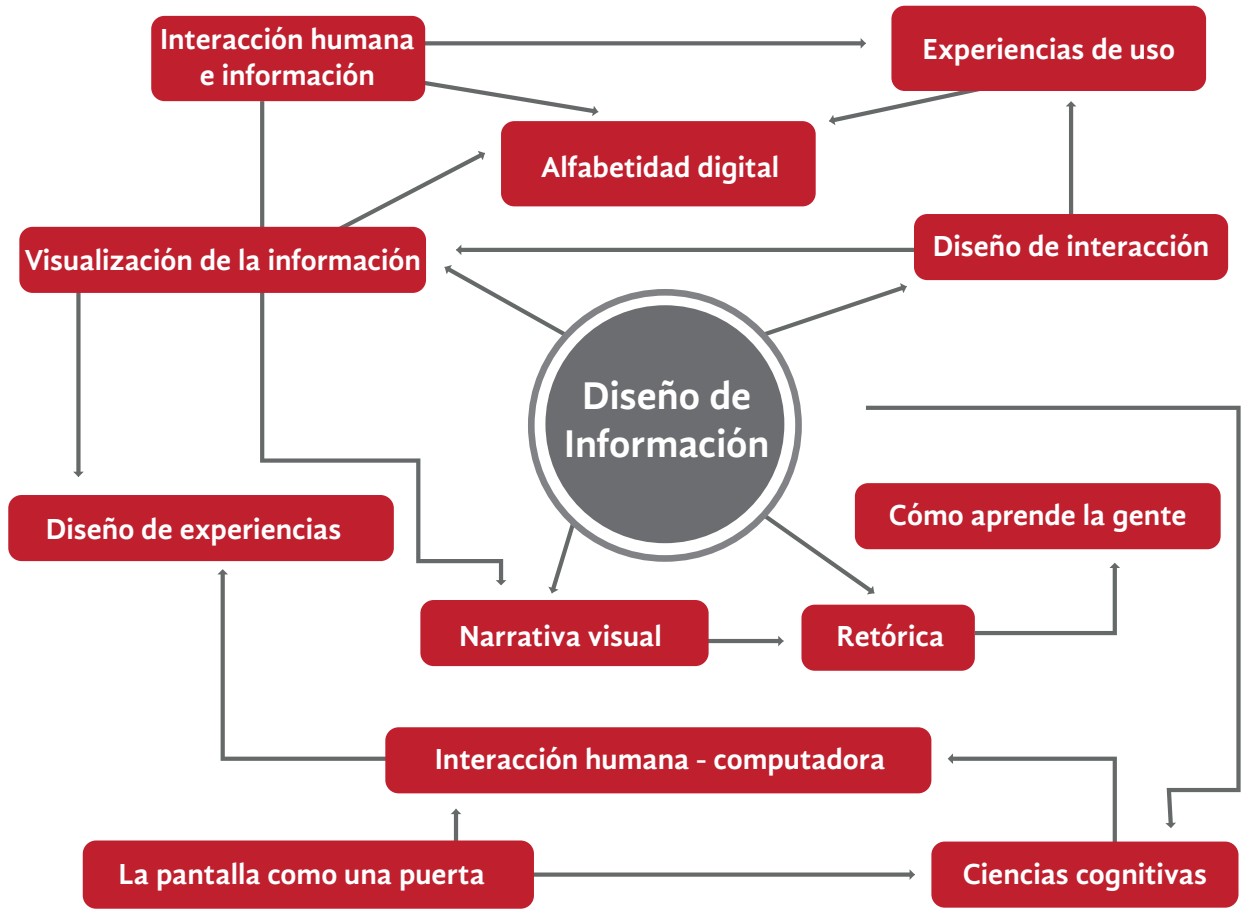

Figura 6. Mapa conceptual con las ideas principales del DI que se conjugan en el MIDIMED.

Fuente: Elaboración propia.

Resultados Al analizar la respuesta de los expertos podemos encontrar presentes las ideas del DI, que son de fuerte resonancia, pues no sólo se mencionan por parte de los participantes, aunque de manera intuitiva, al tener que trabajar con datos e información compleja; sino que cobran importancia 
durante la fase de formación del proyecto, así como durante la ejecución del modelo por el eco que producen.

Por otro lado, los expertos aportaron información relevante durante cada ronda para mejorar las etapas del modelo, a través de su juicio de valoración, el cual confirmó supuestos previos y permitió identificar ideas antes no consideradas (para ver a detalle el comentario de los expertos véase la tabla 2 ).

Tabla 2. Comentarios de los expertos

\section{Sobre las etapas}

«Tienen secuencia, sobre todo, lo llevan a uno de la mano desde el principio, desde decir qué es lo que se quiere con este trabajo hasta la evaluación» (experto 02, entrevista 02, comunicación personal, 23 de abril de 2012).

«Están bien estructuradas. Se hizo un análisis de que no faltara ni sobrara nada, está completo en ese sentido. Creo que están bien desarrolladas, de manera clara y especificada, de manera que permite seguir un proceso y tener un resultado» (experto 10, entrevista 02, comunicación personal, 13 de julio de 2012).

\section{Sobre el modelo}

«Permite aclarar dudas y nos proporciona todos los elementos necesarios para que el docente se introduzca en el proceso educativo, y vaya desarrollando también estrategias que le van a permitir, a su vez, propiciar la adquisición de habilidades en el estudiante, mismas que le van a servir en su trabajo clínico a futuro, a través del desarrollo del razonamiento clínico, que promueve mucho el modelo, pero este pensamiento, este razonamiento clínico muy sistematizado» (experto 05, entrevista 02, comunicación personal, 14 de marzo de 2012).

«Realmente a ti como profesor sí te ubica para plantearte las preguntas importantes: ¿y ahora cómo voy a enseñarles?, o ¿cómo voy a facilitar el aprendizaje de mis alumnos para que puedan aplicar lo que les estoy enseñando?» (docente experto, entrevista 02, comunicación personal, 17 de enero de 2012).

\section{Acerca del valor del MIDIMED}

«Considero que toda la propuesta, dividida en las cinco etapas que mencionas, debiese integrarse en un curso especial previo obligatorio para todos los profesores, con el objeto de unificar criterios de enseñanza y evaluación, no sólo para medicina, sino para toda la Universidad» (experto 01, entrevista 02, comunicación personal, sin fecha).

«Ojalá y este modelo sea promovido y auspiciado por las autoridades de la institución donde se vaya a aplicar para que tengan los profesores la facilidad de elaborar su material de esta manera porque, aun cuando, como comentaba hace un momento, existen en línea, existen software, cada profesor tiene sus propias necesidades en cuanto a la asignatura que imparte y esto lo permite, este modelo le permite a uno crear su propio material, para aplicarlo conforme a la propia experiencia también $y$, sobre todo, utilizando las técnicas de enseñanza que existen en el área médica» (experto 05, entrevista 02, comunicación personal, 14 de marzo de 2012). 


\section{Acerca del valor del MIDIMED}

«Considero que toda la propuesta, dividida en las cinco etapas que mencionas, debiese integrarse en un curso especial previo obligatorio para todos los profesores, con el objeto de unificar criterios de enseñanza y evaluación, no sólo para medicina, sino para toda la Universidad» (experto 01, entrevista 02, comunicación personal, sin fecha).

«Ojalá y este modelo sea promovido y auspiciado por las autoridades de la institución donde se vaya a aplicar para que tengan los profesores la facilidad de elaborar su material de esta manera porque, aun cuando, como comentaba hace un momento, existen en línea, existen software, cada profesor tiene sus propias necesidades en cuanto a la asignatura que imparte y esto lo permite, este modelo le permite a uno crear su propio material, para aplicarlo conforme a la propia experiencia también y, sobre todo, utilizando las técnicas de enseñanza que existen en el área médica» (experto 05, entrevista 02, comunicación personal, 14 de marzo de 2012).

\section{Acerca de la aplicación del modelo}

«Me interesa mucho, desde mi punto de vista como profesor, que los alumnos puedan tener un enfoque muy práctico del conocimiento que están adquiriendo, es decir, que no se quede solamente en la teoría, en el mundo de las ideas, sino que se pueda concretar eso en aspectos prácticos, que ellos puedan aplicarlo casi inmediatamente, ese mismo día con un paciente. Entonces el modelo me ha ayudado en eso. Sí me ha permitido tener un enfoque más práctico en ese sentido. $Y$ lo he sentido en mis clases, en la parte que yo necesitaba... podía yo ya tener una idea más clara de las expectativas que ellos tenían de la clase y de los conocimientos previos. Incluso antes de dar la clase, yo había estado como explorando qué querían aprender en esa clase, ¿no?, yo ya tenía bastante claro cuáles habían sido sus conocimientos previos, a mí como profesor me quedaba muy claro en qué iban a aplicar el conocimiento, entonces, cómo iba a tener que preparar la experiencia de aprendizaje para que ellos estuvieran listos para aplicarlo... los alumnos notaron una diferencia y, nuevamente, es el enfoque muy práctico, y que ellos lo sienten como algo muy valioso, claramente sienten que están mejorando en su camino para convertirse en doctores, están dando pasos en firme y concretos, en los cuales están pudiendo ser mejores doctores, entonces eso es algo muy importante. Sí, el modelo nos ayudó a eso» (docente experto, entrevista 02, comunicación personal, 17 de enero de 2012).

«Creo que la principal virtud o fortaleza es la libertad del modelo para su uso o para su implementación... Una de las cosas que a mí me llama la atención de este modelo es que aquí se pide que el docente desarrolle los materiales, a partir de sus propias necesidades, a diferencia de otros modelos en donde simplemente se le dan los materiales, o se le da lo que tiene que hacer y el docente, pues lo hace, ¿no?, pero no existe esa parte creativa o esa parte de desarrollo que podemos tener con este modelo» (experto 03, entrevista 02, comunicación personal, 08 de junio de 2012). 


\section{En relación con el valor educativo}

«El propósito y la forma de abordarlo me parece excelente, favoreciendo con ello al estudiante y facilitando la enseñanza centrada en el alumno por parte del profesor» (experto 01, entrevista 02, comunicación personal, sin fecha).

«Analizando el modelo, a mí me quedó muy claro que sería la puerta de entrada, haga de cuenta que es como la zona de desarrollo próximo de Vigotsky, que es la brecha que hay entre el nivel básico y el formativo» (experto 02, entrevista 02, comunicación personal, 23 de abril de 2012).

«Este modelo nos permite sistematizar qué es lo que requiere el proceso educativo forzosamente porque, de otra manera, uno obtiene elementos de los diversos cursos que ha tomado y va integrando su material, pero no lo elabora de manera sistematizada. $Y$ con este modelo sí se permite tal situación... Es muy positiva la experiencia, muy positiva. De hecho, a mí me parece que es uno de los elementos que realmente lleva a la adquisición del conocimiento de los muchachos. Les desarrolla competencias, les desarrolla habilidades y, lógicamente, también le facilita a uno el trabajo en el aula» (experto 05, entrevista 02, comunicación personal, 14 de marzo de 2012).

Fuente: Elaboración propia

El proyecto MIDIMED tuvo una gran aceptación y parte de esto se debió a la forma en que se estructuraron las etapas y los puntos que lo conforman, que se integran en una secuencia que facilita su entendimiento y aplicación. En palabras de los miembros del panel de expertos, las cinco etapas que conforman el modelo se perciben planificadas y con una secuencia que facilita su aplicación.

Esto tiene que ver con el DI y con el principio de diseñar interacciones naturales y sencillas, además de que el modelo es un documento comprensible que fácilmente puede ser convertido en acción efectiva. Sobre esto, los expertos opinaron que el modelo, a la vez que guía al docente en el proceso educativo, lo alienta a plantear estrategias adecuadas a sus estudiantes.

A partir de estas observaciones, se puede hacer una lectura del subtexto en donde los profesores reconocen: a) el valor para su práctica, de guías cuya secuencia sea clara y fácil de entender; b) la necesidad de usar apoyos donde se vaya más allá de la mera secuencia «haz esto, luego lo otro, esto más, y listo», donde se aporte un valor ideológico perceptible e integralmente se manejen estrategias para lograr el entendimiento, con el fin de llevar esto a la acción y que repercuta en un empoderamiento que beneficie el aprendizaje del estudiante.

A su vez, la respuesta de los expertos también remite a los principios del di relacionados con establecer una comunicación efectiva, promover el entendimiento y colocar a los usuarios en el centro del proceso de diseño. Al respecto, son especialmente notorias las observaciones que se hace el participante que elaboró material para su clase, la cuales pueden interpretarse como resultado de dos de las ideas más prevalentes del DI: 
la narrativa, y que el DI actúa como una puerta que permite interactuar con el conocimiento previo y producir nuevas ideas.

En la figura 7 se aprecia la arquitectura de información aplicada para la generación de la etapa 1 del MIDIMED. En la parte inferior izquierda se puede leer "Que cada sección sea ocasión de hacer reflexionar al médico» (en azul), y «¿cómo provoco que el estudiante se cuestione la información?» (en blanco). Coincidentemente, estas dos preguntas son las que el experto se hace en su reflexión, con lo que se reafirma que el midimed, más que un modelo estilo «haz esto, luego lo otro, termina con esto», es una guía conceptual que desde sus orígenes bebe su filosofía directamente del Dı (en la creación técnica del modelo) y llega hasta la reacción que produce en las personas que lo implementan.

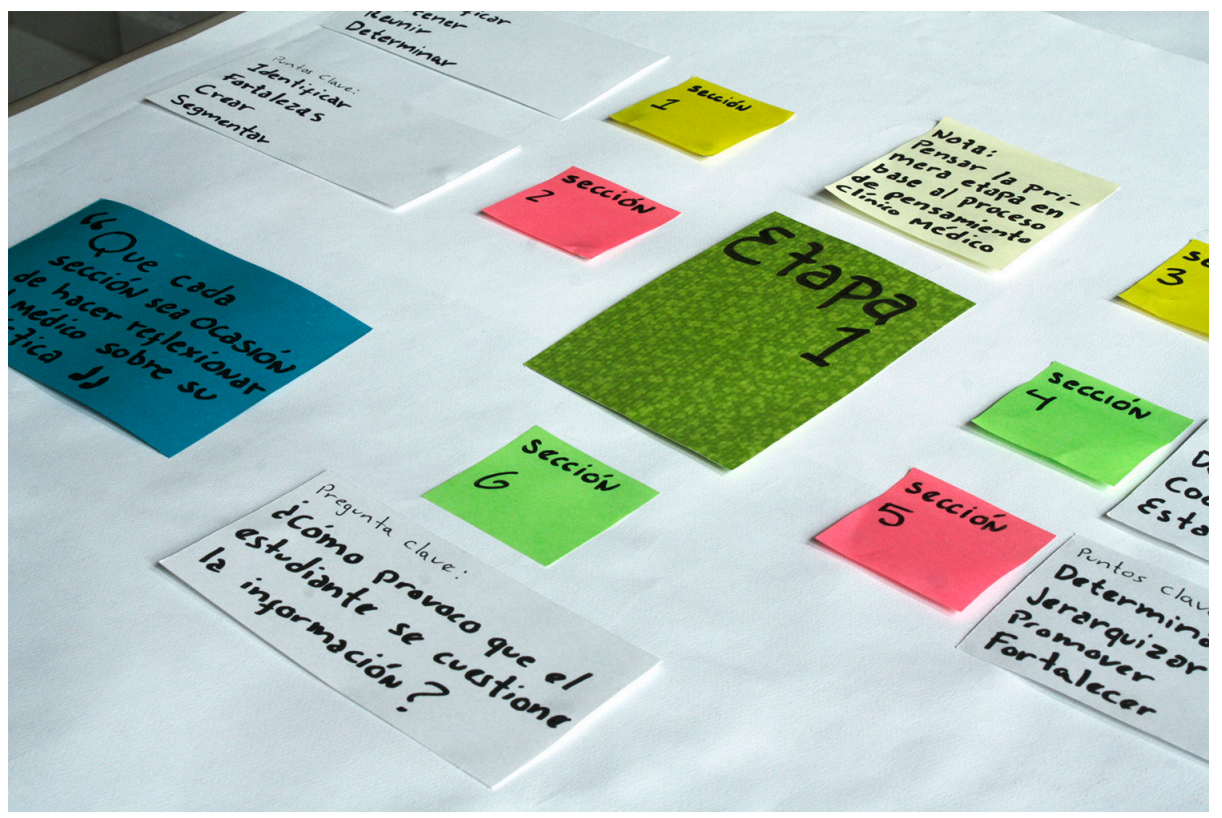

Figura 7. Arquitectura de información estableciendo las ideas centrales de la etapa 1. Fuente: Elaboración propia

Por otro lado, varios miembros del panel señalaron que el proyecto tiene valía, un gran potencial, e incluso que debería hacerse un curso para implementarlo en la capacitación de los profesores de las facultades de medicina, o hasta con un alcance más amplio hacia otras áreas de conocimiento.

Esto remite a las ideas de edificación y conmutatividad acerca de que el conocimiento debe ser para todos y a que el midimed permite aprender con significado mediante una narrativa y una retórica adecuadas.

Además de lo anterior, existen datos alentadores que hablan acerca de cómo, incluso si el modelo se lleva a cabo parcialmente, se pueden obtener resultados benéficos para la enseñanza y la educación, sobre todo en cuestiones de cómo gestionar la información con base en el 
conocimiento previo de los estudiantes, esto producto del enfoque fundamentado en el DI.

En todo lo anterior, nuevamente se aprecia la idea de crear, mediante narrativa y retórica, interacciones que se conviertan en acción efectiva, así como la afirmación de que el actuar del di permite que la acción de la pantalla en medios digitales sea como una puerta que facilita el producir e interactuar con conocimiento valioso.

Otro punto importante a mencionar es que todos los miembros del panel de expertos perciben que los materiales digitales están alineados con las preferencias que tienen los estudiantes y, por tanto, es una manera empática de acercar el conocimiento a ellos. Lo anterior, indiscutiblemente está relacionado con el uso de la narrativa, del contexto sociocultural en que viven las nuevas generaciones, porque se pueden generar ambientes integrados, como lo señalan diversos autores (Monsalve-Lorente y Aguasanta-Regalado, 2020; Sandars y Murray, 2009). Esto se traduce en poner en el centro a las personas.

También los expertos señalaron que el modelo puede ser un vínculo perfecto en la maduración de las habilidades y competencias de los estudiantes, al convertirse en un puente que les permita desarrollarse mejor en sus evaluaciones, estar más capacitados cuando pasen del periodo de preclínica a los siguientes niveles de su aprendizaje, y afrontar situaciones con mayor complejidad. Esto en consonancia con lo que afirman diferentes autores sobre el camino que debe tomar la enseñanza preclínica (Bowe, Voss y Aretz, 2009; Gülpinar, Akman y User, 2009; Scaperotti, Gil, Downs, Jeyakumar, Liu, Chan, Bonner, Kelly, Nosanchuk, Cohen y Jerschow, 2017; Stevens, Holmes, Grainger, Connolly, Prior, Fitzpatrick, O'Neill, Boland, Pawlikowska y Humphreys, 2019). Lo antedicho es resultado de poner en práctica las ideas del DI, en donde se conjugan conceptos cognitivos, de planeación, de comunicación y una profunda vocación de llevar al entendimiento, lo que remite a la acción. En este sentido, ante la facilidad de aplicación del modelo, una vez que se ha entendido de dónde surge esta característica, podemos apoyarnos en lo dicho por los expertos. Los participantes han expresado que el modelo es una guía muy completa que va llevando al profesor paso a paso hasta lograr obtener un material educativo digital relevante. Lo anterior como consecuencia de que el modelo instruccional que se propone es valioso, porque el material digital resultado del proceso facilita reunir lo personal y lo social, entrelazar la teoría con la práctica, dar flexibilidad y control sobre el método, así como ritmo y orden de creación y de acceso a la información, tal como dice la teoría (Mendoza-Villamar y Quiroz-Valencia, 2019; Scaperotti et al., 2017).

Así, se afirma el MIDIMED como un arquetipo centrado en las ideas y en los conceptos principales que abarca el DI, que pone en el centro de su hacer a las personas, lo que da como resultado, para quienes lo utilizan, una empatía y una resonancia con las etapas del modelo. 


\section{Limitaciones}

Es necesario reconocer las limitantes que existen entorno al modelo. Al respecto, debe mencionarse que éste es un estudio empírico e interpretativo, por lo que falta hacer más pruebas de campo que estén enfocadas en el reconocimiento de patrones de la acción del DI, para diferenciar cuándo está actuando y cuándo lo hacen los otros elementos que conforman al midimed (educación y medicina). Además, hay que analizar si estas otras áreas están contribuyendo para enfatizar la acción del DI. Por otro lado, la muestra de expertos fue reducida y selectiva, así que resulta necesario ampliar la población de estudio para tener datos más extensos y profundos.

Conclusiones Este artículo busca mostrar, por un lado, cómo el MIDIMED, en su creación, tuvo una gran influencia del DI, lo que fue clave para fortalecer al modelo, $y$, por otro, que los efectos de la acción del DI permean en los docentes durante la ejecución de los pasos, y que los productos resultantes gestionan la información, apoyando así el proceso educativo de los estudiantes de medicina.

Si sólo se ve a cualquier rama del diseño como acto de praxis, sin un pensamiento ideológico que lo nutra, se obtienen productos que no aportan a mejorar la calidad de vida de las personas. El considerar solamente a la educación no es suficiente, pues ésta necesita ser canalizada y enfocada mediante algo perceptible; tampoco se puede recurrir sólo a la medicina, ya que sin prácticas educativas sólidas únicamente se producen materiales que no tienen un impacto significativo en el aprendizaje de los estudiantes. De esta manera, el catalizador que sirve como puente entre el conocimiento médico, la buena enseñanza de este conocimiento, y el elaborar objetos educativos que realmente enseñen, radica en el uso del DI como mediador de la experiencia educativa.

El DI es un intermediario cuyo objetivo es facilitar la transición entre la intención de hacer algo y el poder hacerlo. Y para lograrlo, se vale del uso de narraciones visuales y de la retórica, que adquieren un matiz de vehículo facilitador del proceso de intercambio de ideas.

El objetivo de este proyecto es que el profesor pueda elaborar sus materiales de aprendizaje digitales, los cuales se presentan ante los aprendices mediante la proyección en algún dispositivo, donde el diseño se encarga de producir la mejor experiencia posible. Cabe aclarar que no se busca convertir al profesor de medicina en un experto del diseño, sino sólo hacerlo consciente de la importancia estratégica que esta área puede aportar a su práctica docente y de que, al usar el MIDIMED, el profesor está implementando prácticas valiosas de diseño. 
La relevancia para el diseño radica en que se demuestra que la acción del DI es capaz de mejorar la práctica de otras disciplinas, además de ser un catalizador que concilia los esfuerzos de diversas áreas del conocimiento, y que esta acción invisible de los principios se convierte en un detonante de procesos conceptuales de alto nivel.

Finalmente, destaca que el diseño de información es una disciplina que puede ser aprovechada por las áreas de ciencias, y en particular en las facultades de medicina, cuya inserción en los procesos de elaboración y planeación de materiales de aprendizaje digitales puede provocar grandes cambios, tanto en la forma como los profesores intervienen en sus cursos, como en la manera en que los estudiantes se involucran, participan e interactúan con la información que se les presenta.

Agradecimientos

A Diana Angélica Martínez Cantero por su apoyo en el desarrollo de las figuras que aparecen en este artículo.

$\checkmark$ Referencias

Ab Latif, R., Dahlan, A., Ab Mulud, Z. y Mat Nor, M. Z. (2017). The Delphi Technique as a Method to Obtain Consensus in Health Care Education Research. Education in Medicine Journal. 9(3), 89-102. https://doi. org/10.21315/eimj2017.9.3.10

Agosto, D. E. (2016). Why Storytelling Matters: Unveiling the Literacy Benefits of Storytelling. Children and Libraries. 14(2), 21-26. https://doi. org/10.5860/cal.14n2.21

Barnes, S. R. (2016). Appearance and Explanation: Advancements in the Evaluation of Journalistic Information Graphics. Journal of Visual Literacy. 35(3), 167-186. https://doi.org/10.1080/1051144X.2016.1278109

Bove, A. A. (2008). Internet-Based Medical Education. Perspectives in Biology and Medicine. 51(1), 61-70.

Bowe, C. M., Voss, J. y Aretz, H. T. (2009). Case Method Teaching: An Effective Approach to Integrate the Basic and Clinical Sciences in the Preclinical Medical Curriculum. Medical Teacher. 31(9), 834-841. https://doi. org/10.1080/01421590902922904

Boye, N., Eberholst, F., Farlie, R., Sørensen, L. B. y Lyng, K. M. (2007). User Driven, Evidence Based Experimental Design; a New Method for Interface Design Used to Develop an Interface for Clinical Overview of Patient Records. Studies in Health Technology \& Informatics. 129(Pt 2), 1053-1057.

Bransford, J. D., Brown, A. L. y Cocking, R. R. (2000). How People Learn: Brain, Mind, Experience, and School: Expanded Edition. Washington: The National Academies Press. https://doi.org/10.17226/9853 
Cárdenas-Figueroa, A. (2019). Teoría de modelos mentales y el constructo experiencia de marca: Un estudio de caso en una escuela de negocios chilena. Estudios Gerenciales. 35(152), 301-312. https://doi.org/10.18046/j. estger.2019.152.3370

Eisner, W. (2004). Graphic Storytelling and Visual Narrative. Estados Unidos: Poor House Press.

Figueiredo, S. (2011). Building Worlds for an Interactive Experience: Selecting, Organizing, and Showing Worlds of Information Through Comics. Journal of Visual Literacy. 30(1), 86-100. https://doi.org/10.1080/23796529.2 011.11674686

Gallardo-Fernández, I. M., Castro-Calvo, De, A. y Saiz-Fernández, H. (2020). Interacción y uso de tecnologías en los procesos de enseñanza y aprendizaje. Educatio Siglo XXI. 38(1), 119-138. https://doi.org/10.6018/educatio.413441

González Di Pierro, C. (2016). Didáctica de las operaciones mentales que intervienen en el proceso de Enseñanza-Aprendizaje. Revista Electrónica Interuniversitaria de Formación del Profesorado. 19(3), 67-75. https://doi. org/10.6018/reifop.19.3.267251

Gülpinar, M. A., Akman, M. y User, I. (2009). A Course, «The Human in Medicine», As an Example of a Preclinical Medical Humanities Program: A Summary of 7 Years. Medical Teacher. 31(10), e469-e476.

Hautz, S. C., Hoffmann, M., Exadaktylos, A. K., Hautz, W. E. y Sauter, T. C. (2020). Digital Competencies in Medical Education in Switzerland: An Overview of the Current Situation. Journal for Medical Education. 37(6), $1-11$.

Horn, R. E. (2000). Information Design: Emergence of a New Profession. En R. Jacobson (Ed.), Information Design. (pp. 15-33). Cambridge: The mit Press.

Jacobson, R. (2000). Why Information Design Matters. En R. Jacobson (Ed.), Information Design. (pp. 2-13). Cambridge: The mit Press.

Liang, H.-N. y Sedig, K. (2009). Characterizing Navigation in Interactive Learning Environments. Interactive Learning Environments. 17(1), 53-75. https://doi.org/10.1080/10494820701610605

Lin, T. S. (2005). Visual Communication for Learning. International Journal of Learning. 12(9), 175-182.

Luna-Gijón, G. y Porras-Hernández, L. H. (2014). Validación de un modelo instruccional centrado en el diseño de materiales digitales de aprendizaje. Investigación en Educación Médica. 3(11), 123-130. https://doi. org/10.1016/S2007-5057(14)72738-3 
Martínez-Fernández, V.-A. y Mahauad-Burneo, M.-D. (2018). Time As a Constituent Value in Attention Economy. 2018 13th Iberian Conference on Information Systems and Technologies (CISTI). 1-6. https://doi. org/10.23919/CISTI.2018.8399463

McKenna, S., Henry Riche, N., Lee, B., Boy, J. y Meyer, M. (2017). Visual Narrative Flow: Exploring Factors Shaping Data Visualization Story Reading Experiences. Computer Graphics Forum. 36(3), 377-387. https://doi. org/10.1111/cgf.13195

Mendoza-Villamar, R. A. y Quiroz-Valencia, P. (2019). Tecnologías de la información y las comunicaciones más utilizadas por universitarios. 3C TIC: Cuadernos de desarrollo aplicados a las TIC. 8(4), 27-43. https://doi.org/10.17993/3ctic.2019.84.27-43

Miguel-Román, J. A. (2020). La educación superior en tiempos de pandemia: Una visión desde dentro del proceso formativo. Revista Latinoamericana de Estudios Educativos. 50(ESPECIAL), 13-40. https://doi.org/10.48102/ rlee.2020.50.ESPECIAL.95

Monsalve-Lorente, L. y Aguasanta-Regalado, M. E. (2020). Nuevas ecologías del aprendizaje en el currículo: La era digital en la escuela. RELATEC. Revista Latinoamericana de Tecnología Educativa. 19(1), 139-154. https:// doi.org/10.17398/1695-288X.19.1.139

O'Connor, S. L. (2010). Creating Effective Slides. The Clinical Teacher. 7, 247250.

Pavlović, J. y Maksić, S. (2019). Implicit Theories of Creativity in Higher Education: A Constructivist Study. Journal of Constructivist Psychology. 32(3), 254-273. https://doi.org/10.1080/10720537.2018.1477639

Pontis, S. (2019). Making Sense of Field Research. A Practical Guide for Information Designers. Londres: Routledge.

Real-Torres, C. (2019). Materiales Didácticos Digitales: Un recurso innovador en la docencia del siglo xxi. 3C TIC: Cuadernos de desarrollo aplicados a las TIC. 8(2), 12-27. https://doi.org/10.17993/3ctic.2019.82.12-27

Ricardo-Barreto, C., Iriarte-Díazgranados, F., Said-Hung, E., Ballesteros-Cantillo, B., Jabba-Molinares, D., Manotas-Salcedo, E., Salas-Álvarez, D., Peláez-Cárdenas, A., Villa-Agudelo, V., Zapata-Álvarez, S., Aarón-Gonzálvez, M., Choles-Almazo, H., Ordoñez-Villa, M., Vergara-Castilla, E., Chavarro-Jiménez, A. y Astorga-Acevedo, C. (2017). Las Tic en educación superior: Experiencias de innovación. Colombia: Universidad del Norte. https://doi. org/10.2307/j.ctt2050wh0

Sandars, J. y Murray, C. (2009). Digital Storytelling for Reflection in Undergraduate Medical Education: A Pilot Study. Education for Primary Care. 20(6), 441-444. https://doi.org/10.1080/14739879.2009.11493832

Scaperotti, M., Gil, N., Downs, I., Jeyakumar, A., Liu, A., Chan, J., Bonner, J., Kelly, M. S., Nosanchuk, J. D., Cohen, H. W. y Jerschow, E. (2017). Development and Evaluation of a Web-Based Dermatology Teaching Tool 
for Preclinical Medical Students. MedEdPORTAL. 13(10619). https://doi. org/10.15766/mep_2374-8265.10619

Serrano, J. M. y Pons, R. M. (2011). El constructivismo hoy: Enfoques constructivistas en educación. Revista Electrónica de Investigación Educativa. 13(1), 1-27.

Solomonidou, C. (2009). Constructivist Design and Evaluation of Interactive Educational Software: A Research-Based Approach and Examples. Open Education: The Journal for Open and Distance Education and Educational Technology. 5(1), 6-24. https://doi.org/10.12681/jode.9693

Stevens, N. T., Holmes, K., Grainger, R. J., Connolly, R., Prior, A.-R., Fitzpatrick, F., O'Neill, E., Boland, F., Pawlikowska, T. y Humphreys, H. (2019). Can e-learning Improve the Performance of Undergraduate Medical Students in Clinical Microbiology Examinations? BMC Medical Education. 19(1), 408. https://doi.org/10.1186/s12909-019-1843-0

Tarkhova, L., Tarkhov, S., Nafikov, M., Akhmetyanov, I., Gusev, D. y Akhmarov, R. (2020). Infographics and Their Application in the Educational Process. International Journal of Emerging Technologies in Learning (IJET), 15(13), 63-80. https://doi.org/10.3991/ijet.v15i13.14647

Udsen, L. E. y Jørgensen, A. H. (2005). The Aesthetic Turn: Unraveling Recent Aesthetics Approaches to Human-Computer Interaction. Digital Creativity. 16(4), 205-216. https://doi.org/10.1080/14626260500476564

Uribe, M. F. (2019). La forma de la información, una gramática que busca facilitar la toma de decisiones al usar tecnología. Revista Internacional de Tecnología, Conocimiento y Sociedad. 7(1), 47-57. https://doi.or$\mathrm{g} / 10.18848 / 2474-588 \mathrm{X} / \mathrm{CGP} / \mathrm{v} 07 \mathrm{i} 01 / 47-57$

Vernon, W. (2009). The Delphi Technique: A Review. International Journal of Therapy and Rehabilitation. 16(2), 69-76. https://doi.org/10.12968/ ijtr.2009.16.2.38892

Visocky O'Grady, J. y Visocky O'Grady, K. (2008). The Information Design Handbook. Estados Unidos: HOW Books.

Wurman, R. S. (2001). Information Anxiety 2. Estados Unidos: QUE.

Xu, A., Zare, H., Dai, X., Xiang, Y. y Gaskin, D. J. (2019). Defining Hospital Community Benefit Activities Using Delphi Technique: A Comparison between China and the United States. PLOS ONE. 14(11), e0225243. https://doi. org/10.1371/journal.pone.0225243 


\section{Sobre el autor Gerardo Luna Gijón}

Doctor en Educación de las Ciencias, Ingenierías y Tecnologías, es profesor de tiempo completo del Colegio de Diseño Gráfico en la Benemérita Universidad Autónoma de Puebla, México. Especialista en Diseño de Información, ha trabajado en diseño editorial y como consultor de UX. Su interés por la manera en que el diseño ayuda a empoderar otras disciplinas, y particularmente en la comunicación de la ciencia, lo ha llevado a la infografía científica, donde enfatiza el papel de la narrativa como medio para acercar el conocimiento a las personas. 